\title{
Application of 3D printing technology to thoracic wall tumor resection and thoracic wall reconstruction
}

\author{
Yi Wu ${ }^{1 \#}$, Na Chen ${ }^{1 \#}$, Zhou Xu ${ }^{1}$, Xiaoqin Zhang ${ }^{1}$, Li Liu ${ }^{1}$, Chunling Wu ${ }^{2}$, Shaoxiang Zhang ${ }^{1}$, Yan Song ${ }^{1}$, \\ Tao $\mathrm{Wu}^{3}$, Hongxiang $\mathrm{Liu}^{3}$, Meng Tang ${ }^{3}$, Wei $\mathrm{Wu}^{3}$ \\ ${ }^{1}$ Institute of Digital Medicine, Biomedical Engineering College, Third Military Medical University, Chongqing 400038, China; ${ }^{2}$ Institute of Medical \\ Imaging, Biomedical Engineering College, Third Military Medical University, Chongqing 400038, China; ${ }^{3}$ Department of Cardiothoracic Surgery, \\ Southwest Hospital, Third Military Medical University, Chongqing 400038, China \\ Contributions: (I) Conception and design: Y Wu, W Wu; (II) Administrative support: Y Wu, W Wu; (III) Provision of study materials or patients: \\ T Wu, H Liu, M Tang, W Wu; (IV) Collection and assembly of data: T Wu, H Liu, N Chen, Z Xu, X Zhang, L Liu, C Wu; (V) Data analysis and \\ interpretation: N Chen, Z Xu, X Zhang, L Liu, C Wu; (VI) Manuscript writing: All authors; (VII) Final approve of manuscript: All authors. \\ \#These authors contributed equally to this work. \\ Correspondence to: Wei Wu. Department of Cardiothoracic Surgery, Southwest Hospital, Third Military Medical University, No. 30 of Gaotanyan \\ Street, Chongqing 400038, China. Email: wuweiyahoo@sohu.com.
}

Background: Thoracic wall tumors can leave large defects in the thoracic wall after tumor resection. Currently, the shape of the materials commonly used for thoracic wall repair, including dacron mesh and titanium alloy mesh, cannot readily conform to the shapes of defect sites. In this study, we aimed to retrospectively review and evaluate the outcomes of applying three-dimensional (3D) printing technology in assisting in thoracic wall tumor resection and thoracic wall construction.

Methods: Six patients with thoracic wall tumors underwent thin-slice CT scanning. We 3D reconstructed pleural tumors and adjacent structures with Amira software and 3D printed them. Preoperative simulation, surgical rehearsal, and surgical planning were performed, and $3 \mathrm{D}$ conformal titanium plates were created based on 3D reconstruction models and sutured to the defect sites of the thoracic wall. We also retrospectively reviewed 10 patients who underwent this surgery with conventional methods. All of the demographic data, clinical data, and laboratory findings (non-normally distributed variables) were compared between these two groups.

Results: 3D reconstructions of the tumors and their adjacent structures were successfully performed, and $3 \mathrm{D}$ printing physical models and conformal titanium plates were also successfully obtained. The plate afforded accurate matching, less bleeding, fewer postoperative complications, and less pain.

Conclusions: This 3D printing technology can aid in preoperative rehearsal, surgical planning, and the manufacturing of 3D implants. The 3D titanium plate has such advantages over traditional implants as having good fit and hardness, improving the surgical accuracy and curative effect, and reducing complications, such as bleeding and pain.

Keywords: 3D printing; 3D visualization; thoracic tumor resection; thoracic wall reconstruction

Submitted Aug 19, 2018. Accepted for publication Nov 15, 2018.

doi: $10.21037 /$ jtd.2018.11.109

View this article at: http://dx.doi.org/10.21037/jtd.2018.11.109

\section{Introduction}

Thoracic wall tumors originate from such tissues as thoracic wall bones, muscles, blood vessels, and nerves (1). These common thoracic tumors erode the thoracic wall and muscle. Thoracic surgery is often performed to resect thoracic wall tumors and their adjacent thoracic wall structures, but this often produces large defects in the thoracic wall, thoracic wall abnormalities, thoracic wall 
collapse, abnormal breathing, thoracic wall necrosis, and other complications (2). Polyester mesh and titanium alloy mesh are commonly used to repair these thoracic wall defects. However, polyester mesh has limited hardness, and conventional titanium plates are not suitable for the shape of damaged thoracic walls, creating difficulties for repair and giving rise to postoperative complications, such as pain, bleeding, and restricted respiratory movement.

In recent years, 3D printing has become widely used in the medical field, especially in surgical simulation, preoperative rehearsal, and the development of auxiliary surgical tools (3-8). Due to controversy regarding the hardness, stability, and quality of $3 \mathrm{D}$-printed products, there are still few reports regarding the use of $3 \mathrm{D}$-printed implants in the human. In addition, reports regarding the use of $3 \mathrm{D}$ printing technology in thoracic surgery remain limited, and it still remains unclear whether this technology can improve the accuracy and effect of surgery and reduce the rate of surgical complications.

We selected 6 patients who required thoracic wall tumor resection and thoracic wall reconstruction. We created $3 \mathrm{D}$ reconstructions of the thoracic walls and adjacent structures using computer tomography (CT) data, followed by $3 \mathrm{D}$ printing. We then simulated and rehearsed the surgery. Finally, we created conformal titanium plates for thoracic wall reconstruction. The clinical records of these 6 patients were retrospectively analyzed and also to those of patients who underwent surgery with conventional materials.

\section{Methods}

\section{Patient selection}

The patients were selected from January 2016 to January 2018. Inclusion criteria were as follows: (I) thoracic tumor; (II) requirement for resection of part of the thoracic wall and thoracic wall muscles and resultant postoperative thoracic wall defects; (III) otherwise good health. Exclusion criteria were as follows: (I) tuberculosis or inflammatory lesions; (II) Diameter of the excision range under than $5 \mathrm{~cm}$.

Six patients were selected as the $3 \mathrm{D}$-printing group, including 3 males and 3 females, with an average age of (43.2 \pm 23.8 ) years (range, $10-68$ years) (Table 1 ). Ten patients were selected as the conventional methods group, including 6 males and 4 females, with an average age of $(42.9 \pm$ $14.3)$ years (range, $14-64$ years) (Table 2).

\section{Strategy for 3D-printing group}

\section{Three-dimensional reconstruction}

We imported the patient's Dicom format image data using Amira software (http://www.amiravis.com, version 5.2.2) to segment the patient's tumor, lung, sternum, rib, thoracic vertebrae, costal cartilage, lung, liver, skin, and other structures. The lungs, sternum, ribs, thoracic vertebrae, and skin were automatically segmented. The costal cartilage, liver, and other structures were semi-automatically segmented and $3 \mathrm{D}$ reconstructed. Then, the model was smoothed and simplified, followed by $3 \mathrm{D}$ visualization and measurement. Finally, STL format files were generated for $3 \mathrm{D}$ printing.

\section{D printing}

We used stereolithography (SLA) (Formlabs Form2) for 3D digital model printing with liquid photosensitive resin with a maximum print size of $145 \times 145 \times 175 \mathrm{~mm}^{3}$ and a print layer thickness of $0.02-0.1 \mathrm{~mm}$. The larger models were printed using the MakerBot ReplicatorTM 2X, with a maximum print size of $400 \times 300 \times 200 \mathrm{~mm}^{3}$ and a print layer thickness of $0.1-0.4 \mathrm{~mm}$. After 3D printing was completed, the $3 \mathrm{D}$ printed models were simplified, and their surfaces were smoothed. The tumor, bone, and cartilage tissue structures in the model were colored using spraypaint and acrylic paint.

\section{Surgical planning}

The 3D model constructed using Amira software was used to design the surgical approach and method of operation. The surgical resection line of the tumor was drawn, an outline at least $2.5 \mathrm{~cm}$ around the malignant tumor (9). Then, the 3D implant plate was designed to perfectly match the excision margin. Simultaneously, according to the $3 \mathrm{D}$ printing model, the 3D morphology and spatial adjacency relationship of the tumor were re-observed, and the plans for tumor resection and thoracic wall reconstruction were applied.

\section{D conformal titanium plate production}

Using to the $3 \mathrm{D}$ printing model, the homemade CNC and EDM equipment was used to produce point-to-point extrusion. The operating engineer set the processing parameters for one-time shaping based on the patient's condition. During the process, a 3D engraving machine was 


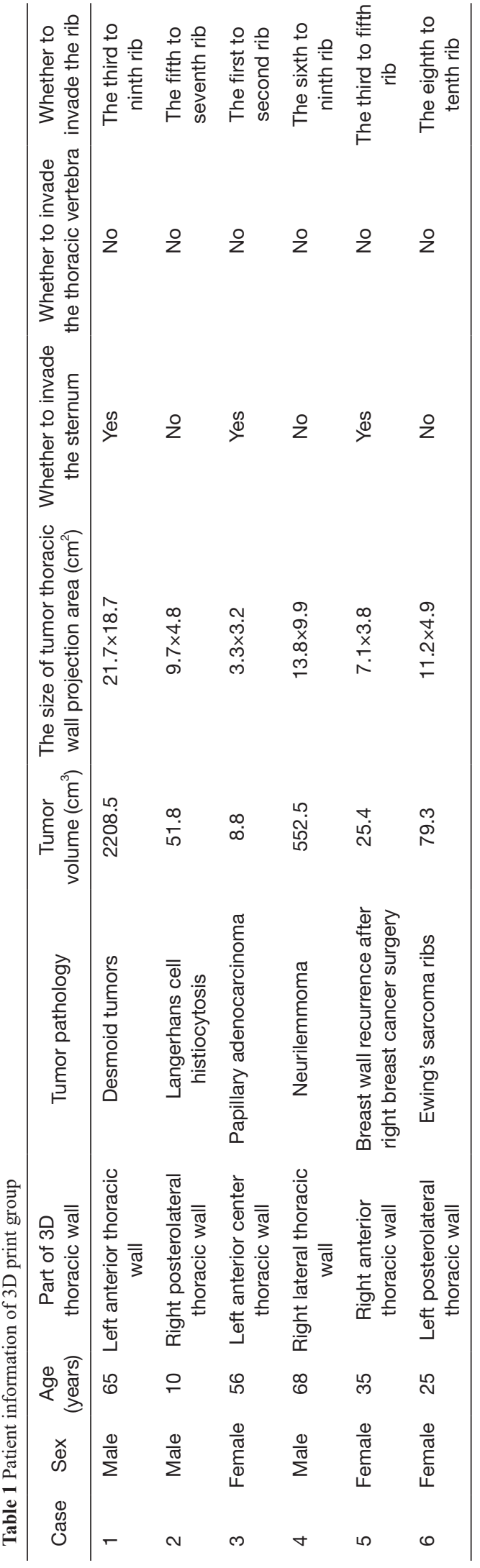

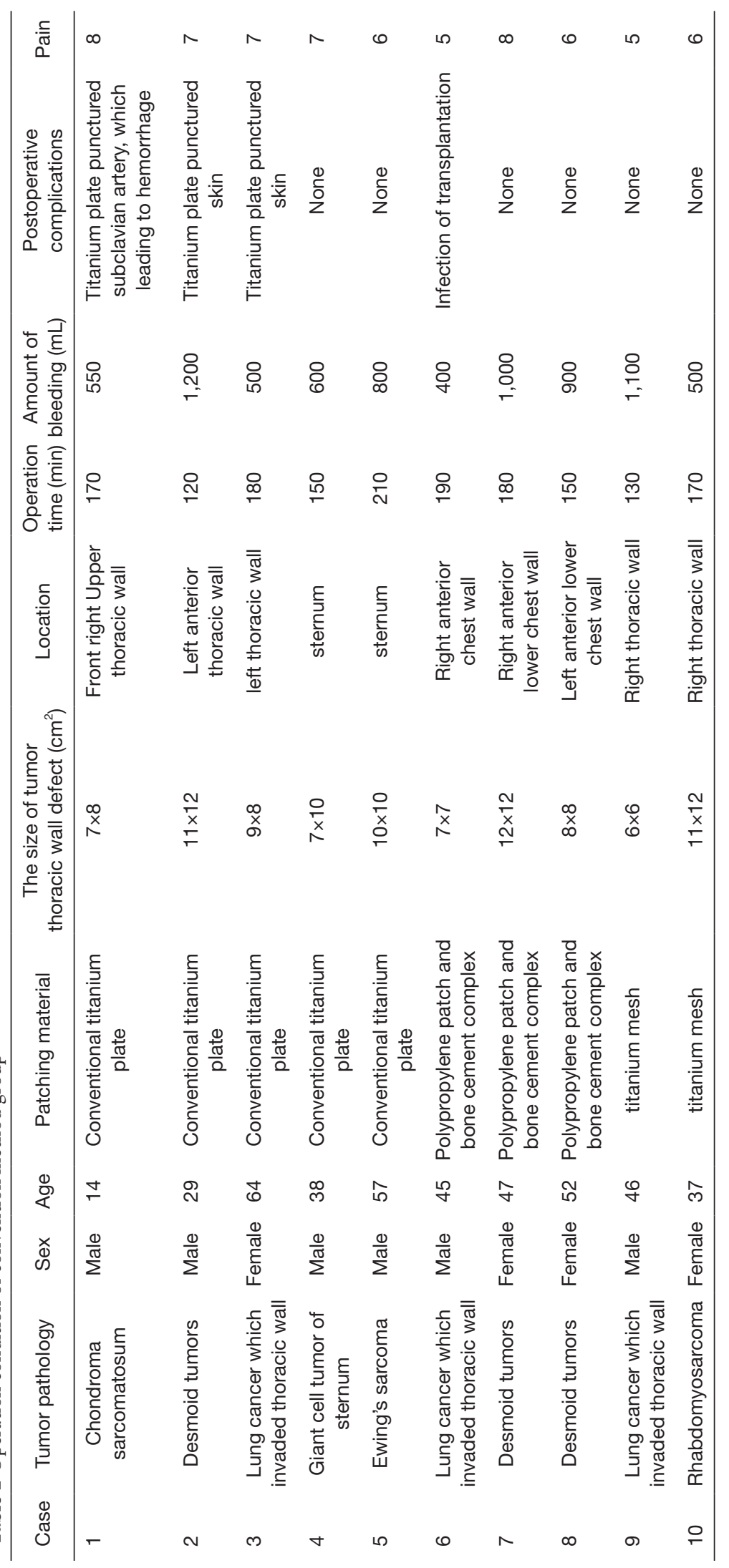




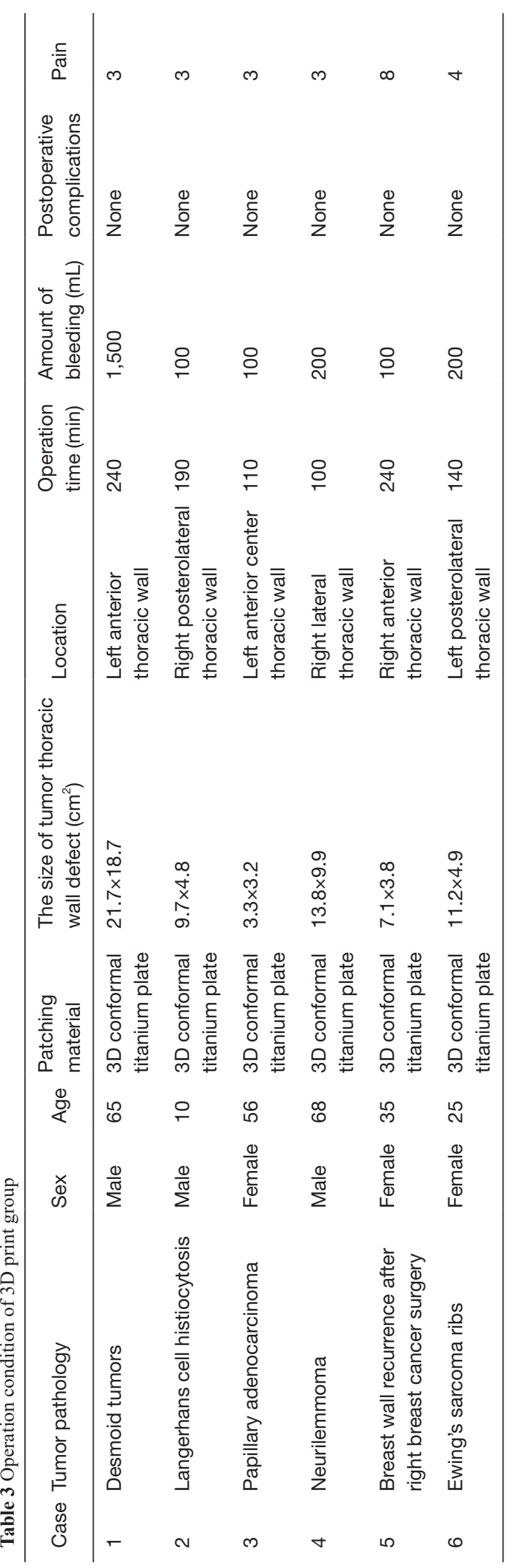

used to create a 1:1 3D conformal titanium plate, followed by inspection, disinfection, and packaging.

\section{Surgery}

Before surgery, we calculated each patient's pulmonary function. We performed thoracic wall tumor resections in the patients. The excision range included the entire thoracic wall tumor, tumor-infiltrated ribs, costal cartilage, and intercostal muscle. The resection margin was at least $2.5 \mathrm{~cm}$ around the tumor. In the surgical margin, we collected tissue from the cutting edge and made frozen pathological sections to check for metastasis and so avoid incomplete excision. We located the $3 \mathrm{D}$ conformal titanium into the thoracic wall defect and fixed it to the thoracic wall with screws (Table 3).

\section{Fifteen days follow-up}

We performed thin-layer high-resolution CT scan as a recheck 15 days after surgery, with a scan image resolution of $512 \times 512$ and a layer spacing of $0.7 \mathrm{~mm}$. Then, 3D reconstruction of the $3 \mathrm{D}$ conformal titanium plate and the thoracic wall bone was performed, and the titanium plate insertion was re-checked. We also calculated the pulmonary function of the patients in the $3 \mathrm{D}$ group.

\section{Strategy for the conventional method group}

We performed thoracic wall tumor resections in the 10 patients. The excision range included the entire thoracic wall tumor, tumor-infiltrated ribs, costal cartilage, and intercostal muscle. The resection margin was at least $2.5 \mathrm{~cm}$ around the tumor. We inserted the conventional titanium plate, titanium mesh, or polypropylene patch and bone cement complex into the thoracic wall defect and fixed it to the thoracic wall with screws (Table 2).

\section{Ninety days of follow-up}

In a further follow-up after 3 months, the patients were reexamined by the surgeon, which included pain and CT exams.

\section{Statistical analysis}

SPSS 22.0 statistical software was used for statistical analysis. Data were expressed as the frequency and percentage for categorical variables and the mean \pm standard deviation for continuous variables or the median and inter 

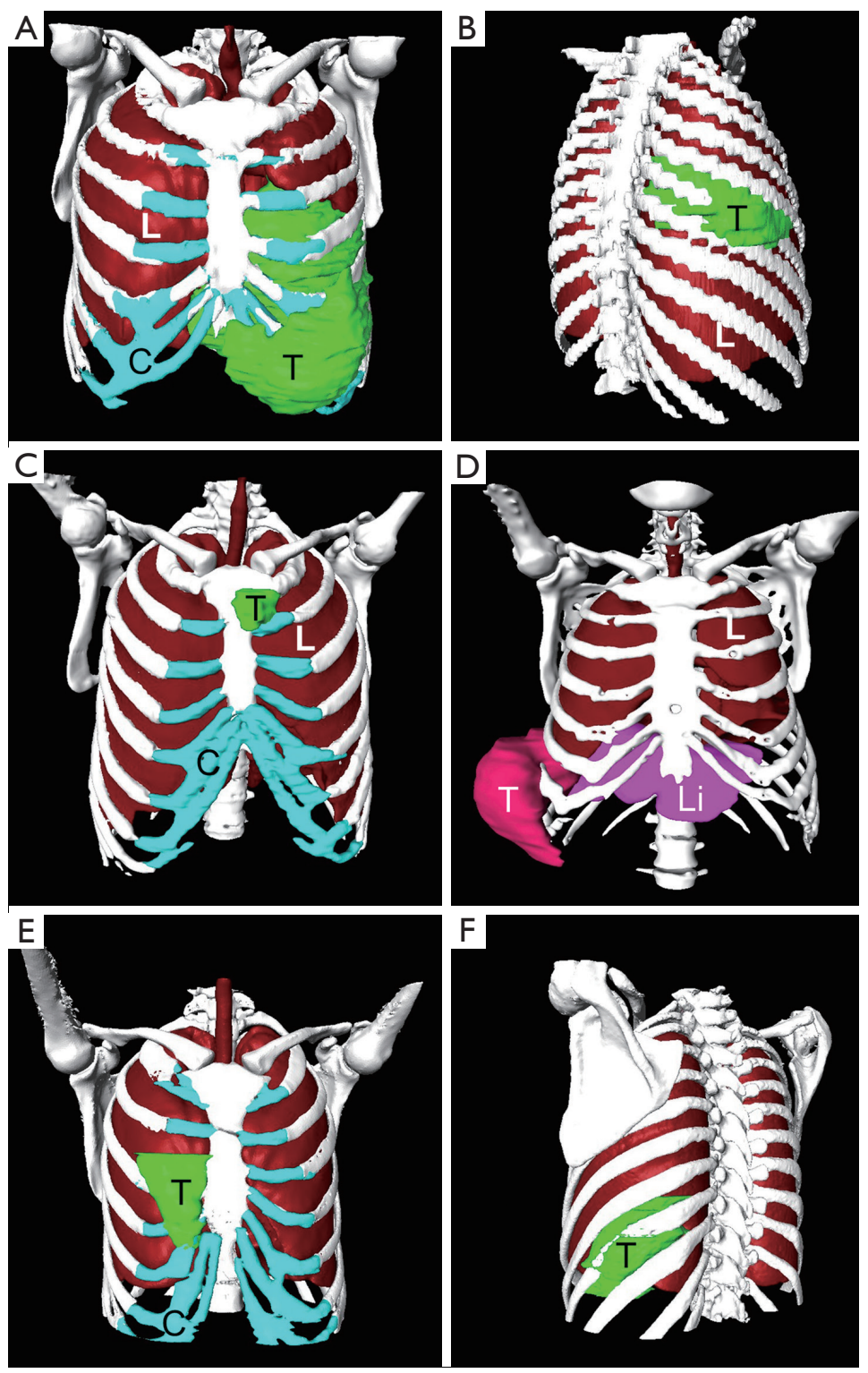

Figure 1 Tumors and adjacent structures of the 6 surgical patients. (A) 3D reconstruction images of aggressive fibroma and adjacent structures for case 1; (B) 3D reconstruction images of Langerhans cell histiocytosis tumor and adjacent structures for case 2; (C) 3D reconstruction images of papillary adenocarcinoma and adjacent structures for case 3; (D) 3D: reconstruction images of neurovascular and adjacent structures for case 4; (E) 3D reconstruction images of right breast cancer and adjacent structures for case 5; (F) 3D reconstruction images of rib Ewing's sarcoma and adjacent structures for case 6. T: tumor; C: cartilage; L: lung; Li: liver.

quartile range (IQR). All demographic data, clinical data and laboratory findings (non-normally distributed variables) were compared between the two groups using the Wilcoxon signed rank test and Mann-Whitney $U$ test for other variables. To compare the proportions of patients, $\chi^{2}$ test or Fisher exact test was performed. All statistical tests were two-sided and significance was defined as $\mathrm{P}<0.05$.

\section{Results}

We successfully constructed 3D digital models of thoracic tumors and their adjacent structures and reconstructed thoracic walls. The adjacent structures included pulmonary blood vessels, lungs, bronchi, ribs, sternum, and costal cartilage (Figure 1). The tumor volumes ranged from 8.8- 

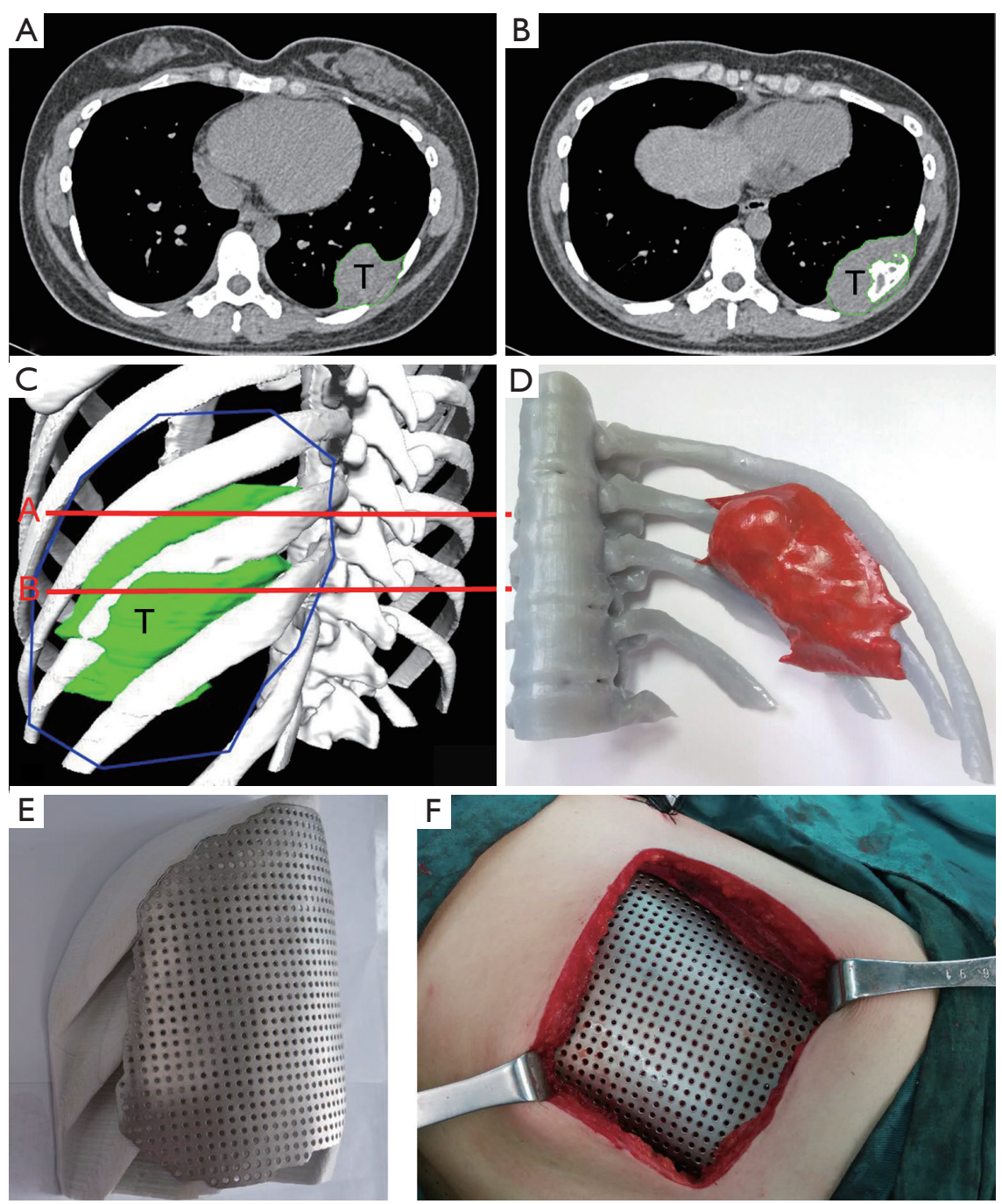

Figure 2 3D reconstruction images, titanium plate design, intraoperative photographs and postoperative CT images of giant desmoid tumors and adjacent structures. (C) 3D reconstruction images of giant desmoid tumors (left front view) and position of the transverse sections in panels (A-B); (D) a 3D conformal titanium plate was successfully implanted in the patients' body; (E) 3D conformal titanium plate design; (F) CT images at 2 weeks after operation. T: tumor.

2,208.5 $\mathrm{cm}^{3}$ (Table 1). Case 1 had the largest tumor volume. This tumor protruded into the left anterior and inferior thoracic wall and the sternum (Figures 1A,2). Case 6 was Ewing's sarcoma, which was located in the posteromedial lateral thoracic wall (Figure 1F). Case 4 was neurilemmoma, $552.5 \mathrm{~cm}^{3}$ that protruded substantially into the outer side of the sixth to ninth chest ribs (Figure 1D). Case 5 was breast cancer recurrence, which was located in the third to fifth ribs of the right anterior thoracic wall, with invasion of the sternum (Figure 1E).

Based on the 3D reconstruction model, we successfully $3 \mathrm{D}$ printed the model using a photosensitive resin printer and studied the 3D morphology and the spatial relationships of the tumor. The scope of the surgical resection and the size, area, space location, and shape of the resected thoracic wall were clarified to provide enough information prior to surgery. The $3 \mathrm{D}$ conformal titanium models were successfully designed and created (Figures 3-5).

We successfully completed the thoracic tumor resection and thoracic wall reconstruction. Since the tumor in case 1 was large, diaphragmatic and pericardial repair were performed (Table 3). The recurrent breast cancer patient (case 5) had thoracoabdominal wall flap metastasis; so we performed flap transplantation. The skin was obtained 

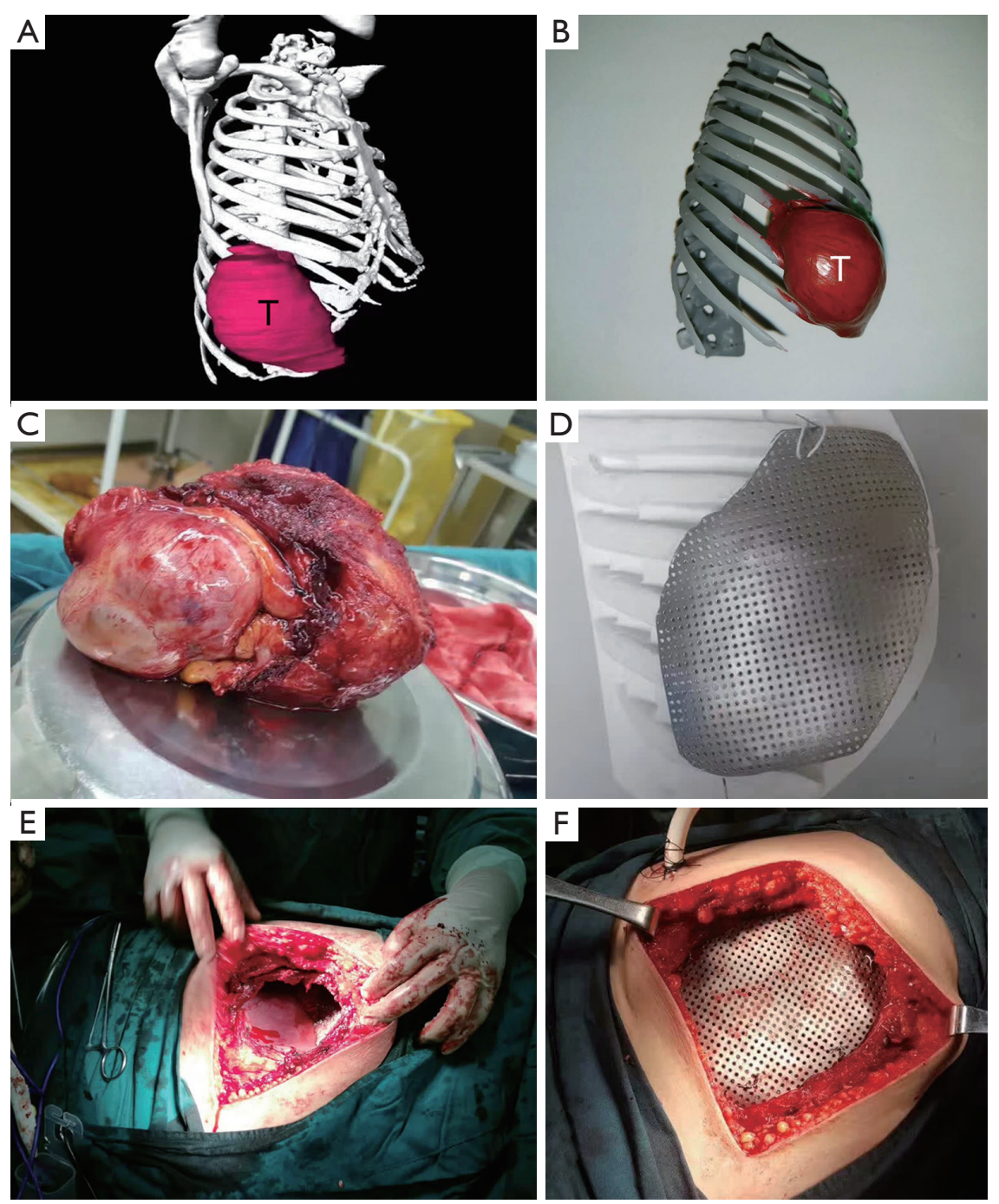

Figure 3 3D reconstruction images, 3D prints and surgical charts of Ewing's sarcoma tumor and adjacent structures. (A) CT cross-sectional image of Ewing sarcoma (A level); (B) CT cross-sectional image of Ewing's sarcoma (B level); (C) 3D reconstructed images and surgical resection area of Ewing's sarcoma and adjacent structures; (D) 3D printing model of Ewing's sarcoma and the corresponding ribs and thoracic vertebra; (E) 3D conformal titanium plate; (F) A 3D conformal titanium plate was successfully implanted in the patient's body. T: tumor.

from the right femur. Although the sizes of the resected tumors were different (Table 1) and the volume of these tumors was relatively large, the $3 \mathrm{D}$-shaped titanium plates were completely consistent with the thoracic wall defect. In 3D-printing group, the surgical bleeding was less, and the patients had good curative effect and recovery (Table 4).

According to the $3 \mathrm{D}$ reconstructed images by CT scan (Figure 4) at 15 days after operation, it was found that titanium plates produced with the assistance of $3 \mathrm{D}$ printing were completely consistent with the thoracic wall defect sites in all 6 cases (Figure 2D). No fixed titanium plate displacement was observed, and patients had good postoperative activity, good respiratory movement, and no abnormal respiratory movements.

According to the CT exam performed 90 days after surgery, in the $3 \mathrm{D}$-printing group, there were no postoperative complications in the $3 \mathrm{D}$-printing group, such as hemorrhage, pneumothorax, fixed titanium plate displacement, metastasis, or abnormal breathing. However, in the conventional methods group, 4 patients showed such complications as infection and puncture of the artery or skin by the titanium plate (Tables 3,4). The post-operation pain 

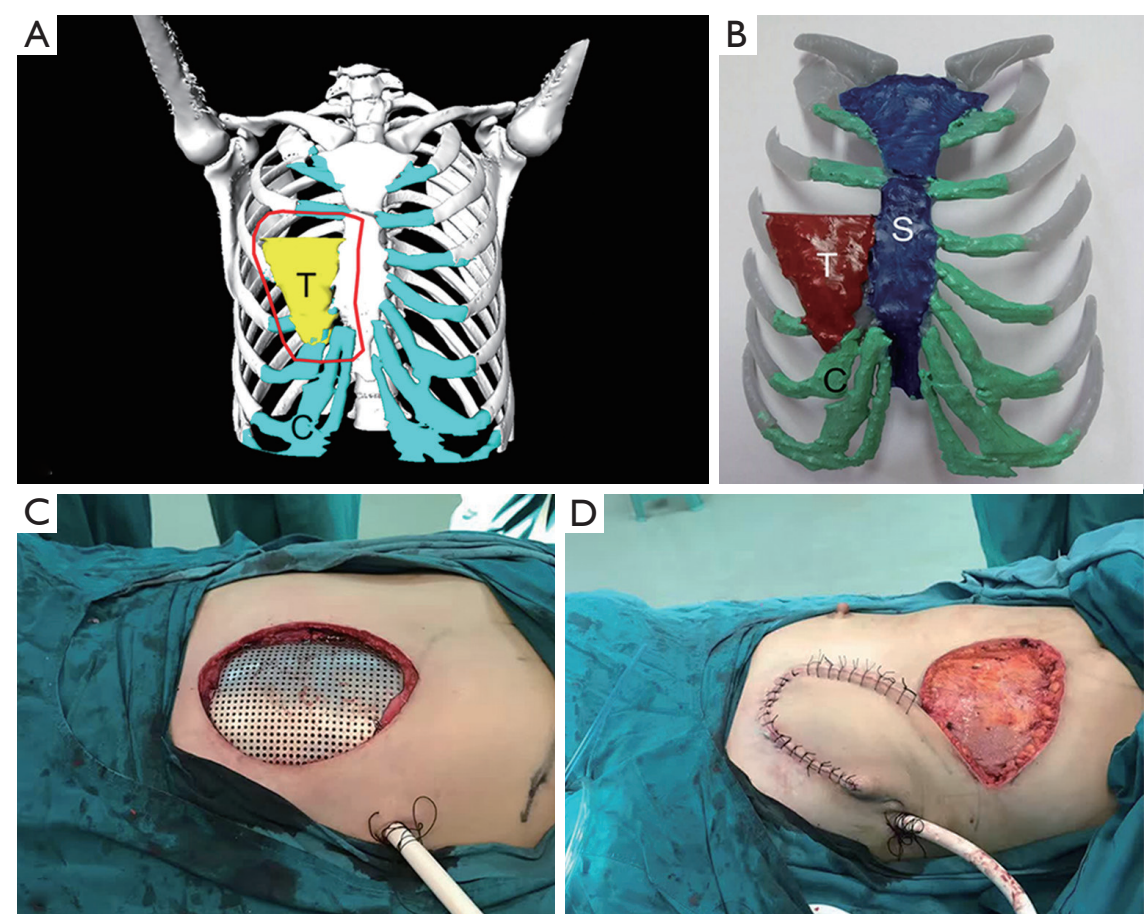

Figure 4 3D reconstruction images, 3D prints and surgical photographs of breast cancer and adjacent structures. (A) 3D reconstruction image of breast cancer (front view); (B) 3D model of breast cancer (front view); (C) A 3D conformal titanium plate was successfully implanted in the patient's body; (D) flap transplantation was performed after 3D titanium plate insertion. T: tumor; S: sternal; C: cartilage.

score was lower than in the conventional methods group (Tables 2,3).

The pulmonary function of the patients in $3 \mathrm{D}$ group showed no significant difference before and after the operation $(\mathrm{P}<0.05)$ (Table S1).

\section{Discussion}

$3 \mathrm{D}$ printing technology is widely used in medicine, including such fields as orthopedics, plastic surgery, maxillofacial surgery, dentistry, and urology (3-8). 3D printing is mainly used for auxiliary diagnosis, surgical design, and surgical rehearsal. Resection of the sternum, rib and cartilage requires thoracic wall reconstruction using a material that permits stabilization of the chest, ensures proper respiratory function, and protects the thoracic organs (10). To date, no treatment scheme has been established for the thoracic wall. There is a general consensus that every malignant tumor should be removed entirely, ideally with $2-5 \mathrm{~cm}$ margins of healthy tissue (11). The choice of chest reconstruction depends on the size of the defect and operator preferences. Defects of $<5 \mathrm{~cm}$ in diameter do not usually require rigid implant reconstruction
$(12,13)$. Therefore, for resection of larger sternal and rib fragments, it is often recommended that they be replaced with homogenous or allogeneic material.

The range of implants used in the thoracic wall is very broad. The most commonly used materials are methyl methacrylate (10), titanium (9) coated with sealed prolene mesh from inside and outside of the thoracic wall, Marlex, polytetrafluoroethylene, and Vicryl. In addition, for the Ley's prostheses in the form of a footprint-shaped titanium plate fixed to the ribs with metal threads, 8 sternum and rib fragments made of $3 \mathrm{D}$-printed titanium $(14,15)$ are also used; however, this 3D technology is expensive, and 3D printing has yet to undergo ethical review. Silicone implants based on patients' chest prints prior to surgery can be used as a less expensive surgical option, but they have the most complications, including seroma formation $(31 \%)$ and lower aesthetic satisfaction (only 69\%). This patient had a severe physical examination (PE) deformity and in retrospect would have been a better candidate for thoracic surgical correction at a young age because the implant was not very hard (16). Autografts of osseous tissue and muscle fragments and allografts of the sternal fragment can be obtained from deceased donors (11). However, these implants are less rigid 
A
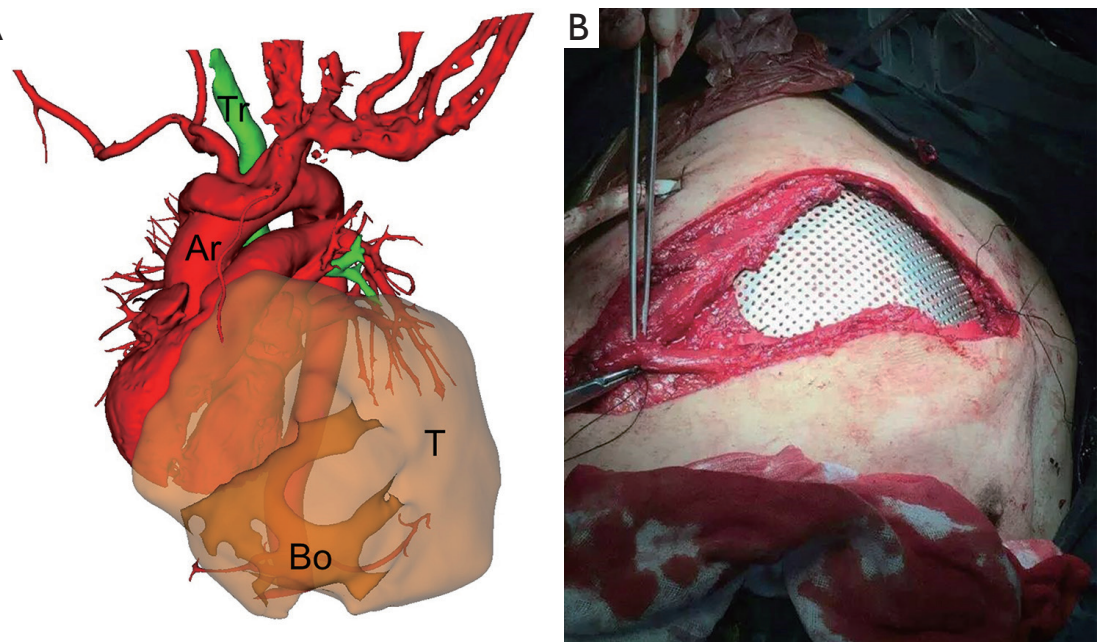

C
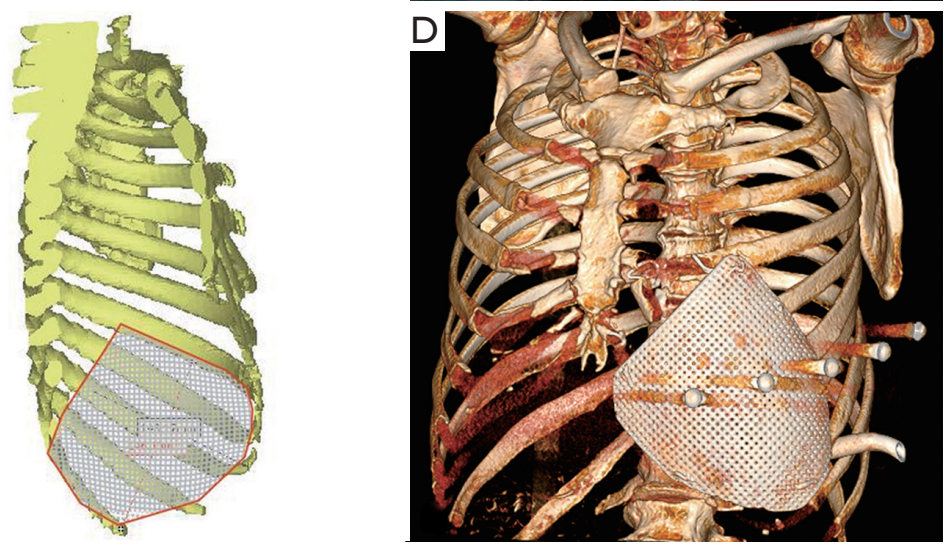

Figure $53 \mathrm{D}$ reconstruction images, titanium plate design, intraoperative photographs and postoperative CT images of giant invasive fibroma and adjacent structures. (A) 3D reconstruction images of giant invasive fibroma (left front view); (B) A 3D conformal titanium plate was successfully implanted in the patients' body; (C) 3D conformal titanium plate design; (D) CT images at 2 weeks after operation. T: tumor; Bo: bone in tumor; Ar: artery; Tr: trachea.

Table 4 Comparison of demographic data and clinical characteristics between the two groups

\begin{tabular}{lccc}
\hline Characteristics & 3D print group & Convention method group & P value \\
\hline Number & 6 & 40 & $10.50[35.00-53.25]$ \\
Mean age & $45.5[21.25-65.75]$ & $6(60 \%)$ & 0.914 \\
Male gender & $3(50 \%)$ & $170[145-182]$ & 1 \\
Operation time $(\mathrm{min})$ & $165[108-240]$ & $700[500-1,025]$ & 0.957 \\
Amount of bleeding $(\mathrm{mL})$ & $150[100-525]$ & 4 & 0.029 \\
Postoperative complications & 0 & $6[5-7]$ & 0.234 \\
Pain & $3[3-5]$ & 0.024 \\
\hline
\end{tabular}


and do not resist stress from respiration, thoracic support, or load-bearing. An implant should be resistant to the forces that act on the skeleton in the chest but pliable enough to adjust it according to the size of the sternum defect, translucent under $\mathrm{X}$-rays, and made of a material that resists bacterial growth (10). Polyethylene appears to be a good material for sternal implants because it is rigid and nonabsorbable and it does not react with bones, soft tissues, or body fluids. It has proper rigidity and resistance but it is radio-opaque on $\mathrm{X}$-rays. It can be fixed to metal plates (titanium, surgical steel) and bones using screws. However, it is not recommended for joints as an endoprosthesis because it is prone to surface abrasion, which can be harmful.

Reconstruction procedures after sternum resection are difficult and are complicated by a significant percentage of cases with complications-estimated between $33 \%$ and $46 \%$. The most often observed complications are those associated with respiratory disorders (16). Other known complications associated with sternum implants are chronic pain, chest deformations, local pleural thickening, and necrosis of pedicle muscle lobes (9), mediastinitis, and loosening of the implant mounting, resulting in instability of the chest. Sternal implant fractures have also been reported (17). A conventional titanium plate is flat and rectangular, and it does not line up with the broken ends of bone defects, does not fit the thorax, shifts easily, and wears through the skin, causing postoperative pain and hematoma. The $3 \mathrm{D}$ printing of titanium alloy implants remains controversial in surgery. Due to their hardness and strength, titanium alloy $3 \mathrm{D}$ implants have yet to be approved by hospital ethics committees and they bring on certain medical risks. Nevertheless, it is feasible to use 3D printing to assist the formation of titanium plates, as the titanium plate prior to $3 \mathrm{D}$ printing has passed FDA certification.

$3 \mathrm{D}$ digital navigation and printing technology are used in tumor resection and reconstruction of the chest in the field of thoracic surgery, which helps surgeons better understand the preoperative thoracic wall tumor site, 3D morphology, invasion range, and spatial adjacency relationships, and helps thoracic surgeons make preoperative surgical plans and conduct surgical rehearsals. The $3 \mathrm{D}$-printingassisted titanium plate completely conforms to the threedimensional shape of the thoracic wall, thereby improving the curative effect of the surgery, shortening the operation time, and substantially reducing the abrasion of the implant on the residual sternum, ribs, thoracic wall muscle, and pleura. No surgical complications, such as bleeding or abnormal breathing, were observed in 6 cases, but in the conventional methods group, 4 patients had such complications as infection and puncture of the skin or artery by the titanium plate. Moreover, the cardiothoracic implants designed by $3 \mathrm{D}$ printing were used to fill the thoracic wall, which played an important role in protecting the contents of the thorax, such as the heart and lungs, and did not involve bearing weight or strenuous exercise. Therefore, the biomechanical requirements of the $3 \mathrm{D}$ conformal titanium plate were lower than those of orthopedic load-bearing parts, rendering the procedure easier.

$3 \mathrm{D}$ conformal titanium plates are not prone to show dislocation or displacement postoperatively. They do not rub against the thoracic wall during normal breathing, thus avoiding local thoracic wall injuries and hematomas. Patients showed little bleeding, except for the 1,500 mL blood loss in one patient with a large chest tumor. Surgical bleeding was only $100-200 \mathrm{~mL}$ in the remaining patients, reducing both blood transfusion costs and the risks of transfusion. The 3D conformal titanium itself is made of titanium alloy. It has a rigid structure, does not deform postoperatively, and resists gravity and tension caused by breathing and tensile forces. The recovery time is greatly shortened, decreasing from two weeks to one week. Moreover, the $3 \mathrm{D}$ conformal titanium plate is not actually a 3D-printed titanium plate and thus is consistent with FDA certification and is easy to implant.

In this study, $3 \mathrm{D}$ printing aided successful thoracic tumor resection and thoracic wall reconstruction in 6 cases, bringing thoracic surgical resection and thoracic wall reconstruction to a new level.

\section{Limitations}

Due to the limited number of procedures, and differences among the tumor resection sites and the size and nature of the tumors, it is difficult to develop surgical criteria for resection and reconstruction of the thoracic wall. We expect to increase the number of surgical procedures in the future to improve $3 \mathrm{D}$ printing in assisting thoracic tumor resection and thoracic wall reconstruction and to develop criteria for the procedure.

\section{Acknowledgements}

Funding: This work was supported by the National Natural Science Foundation of China (No. 31771324; http://www. nsfc.gov.cn/), National Key Research and Development 
Program of China (No. 2016YFC0106403; http://program. most.gov.cn/), Military Youth Science Foundation of China (No. 16QNP100) and Postgraduate Teaching Reform Project in Chongqing (No. yjg183144; http://www.cqjw. gov.cn/).

\section{Footnote}

Conflicts of Interest: The authors have no conflicts of interest to declare.

Ethical Statement: The study was approved by the Ethical Committee of Southwest Hospital (IRB number: KY201846). Informed consent of the operation was obtained from all patients before surgery.

\section{References}

1. Lipinska J, Kutwin L, Wawrzycki M, et al. Chest reconstruction using a custom-designed polyethylene 3D implant after resection of the sternal manubrium. Onco Targets Ther 2017;10:4099-103.

2. Ng CS. Recent and Future Developments in Chest Wall Reconstruction. Semin Thorac Cardiovasc Surg 2015;27:234-9.

3. Anderson PA. Clinical Applications of 3D Printing. Spine 2017;42 Suppl 7:S30-1.

4. Flores RL, Liss H, Raffaelli S, et al. The technique for 3D printing patient-specific models for auricular reconstruction. J Craniomaxillofac Surg 2017;45:937-43.

5. Dupret-Bories A, Vergez S, Meresse T, et al. Contribution of 3D printing to mandibular reconstruction after cancer. Eur Ann Otorhinolaryngol Head Neck Dis 2018;135:133-6.

6. Fiz N, Delgado D, Sanchez X, et al. Application of 3D technology and printing for femoral derotation osteotomy: case and technical report. Ann Transl Med 2017;5:400.

7. Hadeed K, Acar P, Dulac Y, et al. Cardiac 3D printing

Cite this article as: $\mathrm{Wu} \mathrm{Y,} \mathrm{Chen} \mathrm{N,} \mathrm{Xu} \mathrm{Z,} \mathrm{Zhang} \mathrm{X,} \mathrm{Liu}$ L, Wu C, Zhang S, Song Y, Wu T, Liu H, Tang M, Wu W. Application of 3D printing technology to thoracic wall tumor resection and thoracic wall reconstruction. J Thorac Dis 2018;10(12):6880-6890. doi: 10.21037/jtd.2018.11.109 for better understanding of congenital heart disease. Arch Cardiovasc Dis 2018;111:1-4.

8. Kuroda S, Kobayashi T, Ohdan H. 3D printing model of the intrahepatic vessels for navigation during anatomical resection of hepatocellular carcinoma. Int J Surg Case Rep 2017;41:219-22.

9. Haraguchi S, Hioki M, Hisayoshi T, et al. Resection of sternal tumors and reconstruction of the thorax: a review of 15 patients. Surg Today 2006;36:225-9.

10. Lee SY, Lee SJ, Lee CS. Sternum resection and reconstruction for metastatic renal cell cancer. Int J Surg Case Rep 2011;2:45-6.

11. Zhao Y, Peng C, Liu Y, et al. Clinical Study of Allogeneic Graft Reconstruction for Sternum Tumor. Exp Clin Transplant 2016;14:353-7.

12. Weyant MJ, Bains MS, Venkatraman E, et al. Results of chest wall resection and reconstruction with and without rigid prosthesis. Ann Thorac Surg 2006;81:279-85.

13. Lipińska J, Kutwin L, Wawrzycki M, et al. Chest reconstruction using a custom-designed polyethylene 3D implant after resection of the sternal manubrium. Onco Targets Ther 2017;10:4099-103.

14. Aranda JL, Jimenez MF, Rodriguez M, et al. Tridimensional titanium-printed custom-made prosthesis for sternocostal reconstruction. Eur J Cardiothorac Surg 2015;48:e92-94.

15. Anderson CJ, Spruiell MD, Wylie EF, et al. A technique for pediatric chest wall reconstruction using customdesigned titanium implants: description of technique and report of two cases. J Child Orthop 2016;10:49-55.

16. Snel BJ, Spronk CA, Werker PM, et al. Pectus excavatum reconstruction with silicone implants: long-term results and a review of the english-language literature. Ann Plast Surg 2009;62:205-9.

17. Yordanov D, Assenov R, Badarov T, et al. A unique case of fractured titanium implant after sternal resection. J Cardiothorac Surg 2013;8:O77. 
Supplementary

Table S1 Pulmonary function before and after operation

\begin{tabular}{lcccc}
\hline Case & FEV1 before operation $(\mathrm{L})$ & FEV1 after operation $(\mathrm{L})$ & MVV before operation $(\mathrm{L} / \mathrm{min})$ & MVV after operation $(\mathrm{L} / \mathrm{min})$ \\
\hline 1 & 2.2 & 2.0 & 80 & 78 \\
2 & 1.8 & 1.7 & 100 & 97 \\
3 & 2.0 & 2.0 & 90 & 90 \\
4 & 1.9 & 85 & 83 \\
5 & 1.0 & 1.8 & 78 & 77 \\
6 & 1.8 & 1.8 & 102 & 98 \\
\hline
\end{tabular}

